\title{
Nutrient Concentration and Their Uptake and Available Nutrients in Soil Influenced by Irrigation, Mulching and Integrated Nutrient Management in Summer Groundnut
}

\author{
Avinash Chandra Maurya, S.K. Verma ${ }^{*}$, Sushil Kumar and Kairovin Lakra
}

Department of Agronomy, Institute of Agricultural Sciences, BHU, Varanasi (UP) 221 005, India

*Corresponding author

\begin{tabular}{|c|c|}
\hline & A B S T R A C T \\
\hline $\begin{array}{l}\text { K e y w o r d s } \\
\text { Available nutrients, } \\
\text { Irrigation, INM, } \\
\text { Mulching, Nutrient } \\
\text { content and uptake. }\end{array}$ & $\begin{array}{l}\text { An experiment was conducted to assess the effect of irrigation, mulching and integrated } \\
\text { nutrient management on nutrient concentration and their uptake and available nutrients in } \\
\text { summer groundnut during } 2015 \text { and } 2016 \text { at the Department of Agronomy, I.Ag.Sc, BHU, } \\
\text { Varanasi. Results showed that application of irrigation at } 100 \mathrm{~mm} \text { CPE recorded highest N, } \\
\mathrm{K} \text { and S content, available nutrients in soil, kernel and haulm yield. However, irrigation at } \\
60 \mathrm{~mm} \text { CPE recorded significantly the highest P content, NPKS uptake, available nutrients }\end{array}$ \\
\hline Article Info & $\begin{array}{l}\text { in soil, kernel and haulm yield over } 80 \text { and } 100 \mathrm{~mm} \text { CPE. Dust mulch recorded higher } \\
\text { nutrient content and available nutrients in soil whereas, paddy straw mulch recorded }\end{array}$ \\
\hline $\begin{array}{l}\text { Accepted: } \\
17 \text { September } 2017 \\
\text { Available Online: } \\
10 \text { November } 2017\end{array}$ & $\begin{array}{l}\text { maximum NPKS uptake, kernel and haulm yield. Among INM treatments, an application } \\
\text { of } 75 \% \text { NPK }+25 \mathrm{~N} \text { through FYM }+60 \mathrm{~kg} \text { sulphur through gypsum recorded significantly } \\
\text { highest NPKS content and their uptake, available nutrients in soil, kernel and haulm yield } \\
\text { as compared to other INM treatments. }\end{array}$ \\
\hline
\end{tabular}

\section{Introduction}

Groundnut (Arachis hypogaea L.) is the major kharif oilseeds crop of India. India ranks first in the world in respect of area and second in production after China. But the productivity (about $1000 \mathrm{~kg} / \mathrm{ha}$ ) of groundnut is quite low as compared to world average productivity (1500 kg/ha) primarily due to its cultivation in acidic soils with low $\mathrm{N}, \mathrm{P}, \mathrm{Ca}, \mathrm{S}, \mathrm{B}$, inadequate organic matter (Noman et al., 2015), moisture stress at various growth stages irrespective of production environments, irrigation methods, varieties and other cultivation practices (Thiyagarajan et al., 2009). Under irrigated conditions, water use efficiency for this crop is low due to improper irrigation management.
The reduction in yield will be greater if severe stress occur during the critical crop growth stages like flowering and pod formation (Saha and Gunri, 2014). Thus the water management is most important factor because groundnut has specific moisture need due to the unique feature of developing the pods underground (Baliarsingh and Mahapatra, 2014). Proper irrigation scheduling helps the crop to put good crop growth and yield (Thiyagarajan et al., 2010). Application mulch materials can be of great use for conserving moisture, regulating soil temperature, improves infiltration, soil water retention, decreases bulk density and for suppression of weeds during early stages of 
crop growth (Sounda et al., 2006). However, the effect varies with soils, climate, kind of mulch material used and the rate of application. The surface mulch favourably influences the soil moisture regime by controlling evaporation from the soil surface (Patel et al., 2017). Irrigation at critical stages and moisture conservation with mulching practices can increase the land and water productivity along with profitability (Ravisankar et al., 2014).

Groundnut is highly responsive to fertilizer application, although groundnut being a legume is capable of fixing atmospheric nitrogen, it responds to small quantity of nitrogenous fertilizer applied as starter dose (Chaudhary et al., 2015). It is an exhaustive crop and removes large amount of macro and micronutrients from soil. None of the sources of nutrient alone can meet the total plant nutrient need of crop adequately. Hence, integrated use of nutrients from chemical, organic, bio-fertilizer is the most efficient way to supply plant nutrients for sustained crop productivity and improved of soil fertility (Mohapatra and Dixit, 2010 and Chavan et al., 2014). Keeping the above points in view, an experiment was conducted to study the combined effect of irrigation, mulching and INM on nutrient content, nutrient uptake and available nutrients in summer groundnut.

\section{Materials and Methods}

A field study was conducted during the summer season of 2015 and 2016 at Agronomy Farm, BHU, Varanasi (23 ${ }^{\circ} .20^{\prime} \mathrm{N}$, $83^{\circ} .03^{\prime} \mathrm{E}$ and $128.93 \mathrm{~m}$ above mean sealevel). The experimental soil was sandy clay loam with $\mathrm{pH}$ 7.86. The soil was low in available nitrogen (206.9 $\left.\mathrm{kg} \mathrm{ha}^{-1}\right)$ and available phosphorus (17.8 $\left.\mathrm{kg} \mathrm{ha}^{-1}\right)$ and medium in available potassium $(233.1 \mathrm{~kg}$ $\mathrm{ha}^{-1}$ ) and sulphur (15.6 $\left.\mathrm{kg} \mathrm{ha}^{-1}\right)$. Average values for bulk density $1.45 \mathrm{~g} \mathrm{~cm}^{-3}$, particle density $2.62 \mathrm{~g} \mathrm{~cm}^{-3}$, filed capacity $19.6 \%$, permanent wilting point $4.30 \%$ and EC were 0.181

$\mathrm{dSm}^{-1}$. The experiment was conducted in split plot design with twenty four treatment combinations. The treatments consisted of three irrigation regimes $\left(\mathrm{I}_{1}-60 \mathrm{~mm} \mathrm{CPE}, \mathrm{I}_{2}-\right.$ $80 \mathrm{~mm} \mathrm{CPE}$ and $\mathrm{I}_{3}-100 \mathrm{~mm} \mathrm{CPE}$ ) and two mulch treatments $\left[\mathrm{M}_{1}\right.$ - paddy straw mulch and $\mathrm{M}_{2^{-}}$dust mulch] in the main plots and four INM levels $\left(\mathrm{N}_{1}-100 \% \mathrm{NPK}, \mathrm{N}_{2}-75 \% \mathrm{NPK}+\right.$ $25 \% \mathrm{~N}$ through FYM+ $20 \mathrm{~kg} \mathrm{~S}$ through gypsum, $\mathrm{N}_{3^{-}} 75 \% \mathrm{NPK}+25 \% \mathrm{~N}$ through Vermicompost+ $40 \mathrm{~kg} \mathrm{~S}$ through gypsum and $\mathrm{N}_{4}-75 \% \mathrm{NPK}+25 \% \mathrm{~N}$ through FYM $+60 \mathrm{~kg}$ $\mathrm{S}$ through gypsum in sub plots. The experiment was replicated three times. Keeping irrigation frequency constant predetermined quantity of water was applied by using Parshall flume. The entire quantity of nutrients as per treatment was applied in at sowing. Groundnut variety 'HG $37^{\prime}$ was sown at row distance of 30x10 cm manually on 25 March 2015 and 10 March 2016. CPE was worked out from the daily Epan data taken from institute observatory. Irrigation was applied to crop at 60, 80 and $100 \mathrm{CPE}$ as per treatment.

The experiment was repeated on the fixed site in the second year. All the general crop management practices were followed to the crops. The crop was harvested by hand on $29^{\text {th }}$ May, 2015 and 20 ${ }^{\text {th }}$ May, 2016, when about $70 \%$ of haulms were dry. Observations on kernel and haulm yield were recorded at harvest of crop. The soil samples were taken from each plot at $0-15 \mathrm{~cm}$ depth at sowing and after harvesting of groundnut crop for the analysis of nutrient concentration in plant and available nutrients in soil as per the standard procedure. The available nutrients in soil $(\mathrm{kg}$ $\mathrm{ha}^{-1}$ ) were calculated by multiplying the nutrient content and weight of soil $(0-15 \mathrm{~cm})$. Nutrient uptake $\left(\mathrm{kg} \mathrm{ha}^{-1}\right)$ was calculated by 
multiplying their nutrient concentration with crop yield. All the experimental data were analysed statistically to draw a valid conclusion.

\section{Results and Discussion}

\section{Nutrient content}

In groundnut, irrigation regimes and mulching did not affect significantly the total $\mathrm{N}, \mathrm{P}, \mathrm{K}$ and $\mathrm{S}$ contents (Table 1). However, N, K and $\mathrm{S}$ contents decreased with increasing number of irrigation and moisture availability, which was mainly due to dilution effect as a consequence of increased dry matter production. The higher $\mathrm{P}$ content in kernels and haulms was recorded with irrigation at 60 $\mathrm{mm} \mathrm{CPE}$ and in paddy straw mulching. Results are corroborated with the findings of Singh et al., (2006) and Verma et al., (2012). Nutrients concentration in kernels and haulms increased with increasing nutrients level and more so with application of $75 \% \mathrm{NPK}+25 \%$ $\mathrm{N}$ through FYM $+60 \mathrm{~kg} \mathrm{~S}$ through gypsum as compared to other INM treatments. This might be due to increased availability in presence of FYM, vermicompost and the synergy between nutrients. The results corroborate the finding of Arunachalam et al., (2012) and Noman et al., (2016).

\section{Nutrient uptake}

Nutrients uptake by groundnut significantly influenced by irrigation regimes, mulching and INM (Table 2 and 3). Irrigation at 60 CPE and paddy straw mulch recorded maximum $\mathrm{N}, \mathrm{P}, \mathrm{K}$ and $\mathrm{S}$ uptake by kernels, haulms and the total nutrients uptake. Better soil moisture conditions prevailing in these treatments could have facilitated more uptake of nutrients. Researlts are corroborated with the research findings of Verma et al., (2015). In INM treatments, application of $75 \% \mathrm{NPK}+$ $25 \% \mathrm{~N}$ through $\mathrm{FYM}+60 \mathrm{~kg} \mathrm{~S}$ through gypsum recorded significantly highest $\mathrm{N}, \mathrm{P}, \mathrm{K}$ and S uptake followed by $75 \% \mathrm{NPK}+25 \% \mathrm{~N}$ through vermicompost+ $40 \mathrm{~kg} \mathrm{~S}$ through gypsum, $75 \% \mathrm{NPK}+25 \% \mathrm{~N}$ through FYM+ $20 \mathrm{~kg} \mathrm{~S}$ through gypsum and100\% NPK, respectively during both the years. Higher uptake was due to the higher dry matter production and higher nutrient content in different parts of plants, which corroborated the findings of Mohapatra and Dixit (2010) and Yadav et al., 2015. The higher uptake of plant nutrients due to application of the organic manures was reflected in the trend of growth and yield performance of the crop Choudhary et al., (2011) and Patra et al., (2011). Noman et al., (2014) reported the increase in S-uptake from 66.3 to $92.4 \%$ due to application of 20 and $40 \mathrm{~kg} \mathrm{~S} \mathrm{ha}{ }^{-1}$ over control, respectively. Increase in $\mathrm{P}$ uptake was due to increase in $\mathrm{P}$ availability from applied fertilizer and inherent soil source and combined effect of released organic acids and organic anions on decomposition of farmyard manure in acid lateritic soil (Dutta and Mondal, 2006, and Vishwakarma et al., 2012).

\section{Available nutrients}

\section{OC, EC and soil pH}

Effect of irrigation regimes, mulching and INM on $\mathrm{pH}$, organic carbon (OC) and electrical conductivity was found to be nonsignificant (Table 4). However, it was slightly higher under $100 \mathrm{~mm} \mathrm{CPE}$ and with dust mulch. Paddy straw mulch recorded lower $\mathrm{pH}$ and $\mathrm{EC}$ and the OC over dust mulch. This was due to fact that residue when left on soil surface and after decomposition increased the organic carbon which lowers down the electrical conductivity and soil $\mathrm{pH}$ (Monsefi et al., 2016). Amongst INM treatments, 75\% $\mathrm{NPK}+25 \% \mathrm{~N}$ through FYM $+60 \mathrm{~kg} \mathrm{~S}$ through gypsum recorded highest $\mathrm{pH}$, organic carbon and electrical conductivity than other INM treatments. 
Table.1 Effect of irrigation regimes, mulching and integrated nutrient management on $\mathrm{N}, \mathrm{P}$ and $\mathrm{K}$ content in kernel and haulm

\begin{tabular}{|c|c|c|c|c|c|c|c|c|c|c|c|c|c|c|c|c|}
\hline \multirow[t]{2}{*}{ Treatment } & \multicolumn{2}{|c|}{$\begin{array}{l}\mathrm{N} \text { content in } \\
\text { kernel }(\%)\end{array}$} & \multicolumn{2}{|c|}{$\begin{array}{l}\mathrm{N} \text { content in } \\
\text { Haulm }(\%)\end{array}$} & \multicolumn{2}{|c|}{$\begin{array}{l}\mathrm{P} \text { content in } \\
\text { kernel }(\%)\end{array}$} & \multicolumn{2}{|c|}{$\begin{array}{l}\mathrm{P} \text { content in } \\
\text { Haulm }(\%)\end{array}$} & \multicolumn{2}{|c|}{$\begin{array}{l}\mathrm{K} \text { content in } \\
\text { kernel }(\%)\end{array}$} & \multicolumn{2}{|c|}{$\begin{array}{l}\mathrm{K} \text { content in } \\
\text { Haulm }(\%)\end{array}$} & \multicolumn{2}{|c|}{$\begin{array}{l}\mathrm{S} \text { content in } \\
\text { kernel }(\%)\end{array}$} & \multicolumn{2}{|c|}{$\begin{array}{l}\mathrm{S} \text { content in } \\
\text { Haulm }(\%)\end{array}$} \\
\hline & 2015 & 2016 & 2015 & 2016 & 2015 & 2016 & 2015 & 2016 & 2015 & 2016 & 2015 & 2016 & 2015 & 2016 & 2015 & 2016 \\
\hline $\mathrm{I}_{1}$ & 15 & 3.09 & 45 & 1.42 & 0.33 & 0.52 & 7 & 0.115 & 1.05 & 1.00 & 1.17 & 1.15 & 0.186 & 0.175 & 0.117 & .113 \\
\hline $\mathrm{I}_{2}$ & 21 & 3.15 & 1.46 & 1.43 & 0.32 & 0.31 & 0.115 & 0.113 & 1.06 & 1.01 & 1.19 & 1.17 & 0.189 & 0.178 & 0.117 & 0.113 \\
\hline $\mathrm{I}_{3}$ & 324 & 3.18 & 140 & 1.46 & 030 & 0.29 & 0.113 & 0.111 & 1.07 & 1.02 & 1.20 & 1.18 & 0.190 & 0.179 & 0.119 & 0.115 \\
\hline $\mathrm{SEm} \pm$ & 0.05 & 0.04 & 0.03 & 0.04 & 0.03 & 0.04 & 0.002 & 0.003 & 0.03 & 0.02 & 0.04 & 0.05 & 0.003 & 0.004 & 0.002 & 0.002 \\
\hline $\mathrm{CD}(\mathrm{H}$ & NS & NS & NS & NS & NS & NS & NS & NS & NS & NS & NS & NS & NS & NS & NS & NS \\
\hline $\mathrm{M}_{1}$ & 3.16 & 3.10 & 1.46 & 1.43 & 0.33 & 0.32 & 0.114 & 0.112 & 1.05 & 1.00 & 1.18 & 1.16 & 0.190 & 0.179 & 0.117 & 0.113 \\
\hline $\mathrm{M}_{2}$ & 3.24 & 3.18 & 1.48 & 1.45 & 0.31 & 0.30 & 0.116 & 0.114 & 1.06 & 1.01 & 1.19 & 1.17 & 0.191 & 0.180 & 0.119 & 0.115 \\
\hline SEm \pm & 0.03 & 0.03 & 0.02 & 0.02 & 0.02 & 0.03 & 0.002 & 0.003 & 0.02 & 0.02 & 0.03 & 0.03 & 0.002 & 0.003 & 0.002 & 0.002 \\
\hline $\mathrm{CD}(\mathrm{P}=0.05)$ & NS & NS & NS & NS & NS & NS & NS & NS & NS & NS & NS & NS & NS & NS & NS & NS \\
\hline $\mathrm{N}_{1}$ & 3.16 & 3.10 & 1.41 & 1.38 & 0.30 & 0.29 & 0.113 & 0.111 & 1.03 & 0.98 & 1.15 & 1.13 & 0.178 & 0.167 & 0.115 & 0.112 \\
\hline $\mathrm{N}_{2}$ & 3.21 & 3.15 & 1.43 & 1.40 & 0.31 & 0.30 & 0.114 & 0.112 & 1.05 & 1.00 & 1.16 & 1.14 & 0.185 & 0.174 & 0.116 & 0.113 \\
\hline $\mathrm{N}_{3}$ & 3.20 & 3.14 & 1.49 & 1.46 & 0.32 & 0.31 & 0.116 & 0.114 & 1.06 & 1.01 & 1.21 & 1.19 & 0.191 & 0.180 & 0.119 & 0.115 \\
\hline $\mathrm{N}_{4}$ & 3.24 & 3.18 & 1.53 & 1.50 & 0.33 & 0.32 & 0.118 & 0.116 & 1.09 & 1.04 & 1.23 & 1.21 & 0.193 & 0.181 & 0.121 & 0.117 \\
\hline SEm \pm & 0.02 & 0.02 & 0.01 & 0.03 & 0.01 & 0.01 & 0.001 & 0.002 & 0.01 & 0.01 & 0.02 & 0.02 & 0.001 & 0.002 & 0.001 & 0.001 \\
\hline $\mathrm{CD}(\mathrm{P}=0.05)$ & 0.07 & 0.06 & 0.03 & 0.10 & 0.03 & 0.03 & 0.003 & 0.005 & 0.04 & 0.04 & 0.06 & 0.07 & 0.003 & 0.007 & 0.003 & 0.003 \\
\hline
\end{tabular}


Table.2 Effect of irrigation regimes, mulching and integrated nutrient management on N, P and K uptake in grain and straw

\begin{tabular}{|c|c|c|c|c|c|c|c|c|c|c|c|c|c|c|c|c|}
\hline \multirow[t]{2}{*}{ Treatment } & \multicolumn{2}{|c|}{$\begin{array}{l}\mathrm{N} \text { uptake in } \\
\text { Kernel } \\
\left(\mathrm{kg} \mathrm{ha}^{-1}\right)\end{array}$} & \multicolumn{2}{|c|}{$\begin{array}{l}\mathrm{N} \text { uptake in } \\
\text { Haulm } \\
\left(\mathrm{kg} \mathrm{ha}^{-1}\right)\end{array}$} & \multicolumn{2}{|c|}{$\begin{array}{l}\mathrm{P} \text { uptake in } \\
\text { Kernel } \\
\left(\mathrm{kg} \mathrm{ha}^{-1}\right)\end{array}$} & \multicolumn{2}{|c|}{$\begin{array}{l}\text { P uptake } \\
\text { Haulm } \\
\left(\mathrm{kg} \mathrm{ha}^{-1}\right)\end{array}$} & \multicolumn{2}{|c|}{$\begin{array}{l}\mathrm{K} \text { uptake } \\
\text { Kernel } \\
\left(\mathrm{kg} \mathrm{ha}^{-1}\right)\end{array}$} & \multicolumn{2}{|c|}{$\begin{array}{l}\mathrm{K} \text { uptake } \\
\text { Haulm } \\
\left(\mathrm{kg} \mathrm{ha}^{-1}\right)\end{array}$} & \multicolumn{2}{|c|}{$\begin{array}{l}\text { S uptake } \\
\text { Kernel } \\
\left(\mathrm{kg} \mathrm{ha}^{-1}\right)\end{array}$} & \multicolumn{2}{|c|}{$\begin{array}{l}\text { S uptake in } \\
\text { Haulm } \\
\left(\mathrm{kg} \mathrm{ha}^{-1}\right)\end{array}$} \\
\hline & 2015 & 2016 & 2015 & 2016 & 2015 & 2016 & 2015 & 2016 & 2015 & 2016 & 2015 & 2016 & 2015 & 2016 & 2015 & 2016 \\
\hline \multicolumn{17}{|c|}{ Irrigation regimes } \\
\hline $\mathrm{I}_{1}$ & 57.9 & 63.0 & 59.0 & 60.1 & 6.07 & 6.52 & 4.76 & 4.87 & 19.3 & 20.4 & 47.6 & 48.7 & 3.42 & 3.57 & 4.76 & 4.78 \\
\hline $\mathrm{I}_{2}$ & 57.7 & 61.0 & 58.4 & 58.8 & 5.75 & 6.00 & 4.60 & 4.64 & 19.0 & 19.5 & 47.6 & 48.1 & 3.39 & 3.44 & 4.68 & 4.64 \\
\hline $\mathrm{I}_{3}$ & 55.6 & 59.2 & 58.3 & 58.3 & 5.15 & 5.40 & 4.42 & 4.44 & 18.4 & 19.0 & 46.9 & 47.2 & 3.26 & 3.33 & 4.65 & 4.60 \\
\hline $\mathrm{SEm} \pm$ & 0.72 & 1.04 & 0.19 & 0.37 & 0.17 & 0.27 & 0.07 & 0.10 & 0.15 & 0.15 & 0.27 & 0.80 & 0.06 & 0.06 & 0.03 & 0.03 \\
\hline $\mathrm{CD}(\mathrm{P}=0.05)$ & 2.13 & 3.11 & 0.58 & 1.09 & 0.51 & 0.78 & 0.20 & 0.29 & 0.45 & 1.21 & 0.82 & 2.39 & 0.18 & 0.19 & 0.10 & 0.09 \\
\hline \multicolumn{17}{|l|}{ Mulch } \\
\hline $\mathrm{M}_{1}$ & 57.7 & 63.6 & 58.8 & 60.6 & 5.70 & 6.10 & 4.61 & 4.75 & 19.3 & 20.5 & 47.3 & 49.2 & 3.43 & 3.63 & 4.73 & 4.79 \\
\hline $\mathrm{M}_{2}$ & 56.4 & 58.5 & 58.5 & 57.8 & 5.62 & 5.84 & 4.57 & 4.55 & 18.5 & 18.6 & 47.3 & 46.7 & 3.28 & 3.27 & 4.59 & 4.69 \\
\hline SEm \pm & 0.41 & 1.03 & 0.09 & 0.33 & 0.14 & 0.23 & 0.06 & 0.07 & 0.12 & 0.37 & 0.24 & 0.71 & 0.04 & 0.05 & 0.03 & 0.02 \\
\hline $\mathrm{CD}(\mathrm{P}=0.05)$ & 1.22 & 3.08 & 0.26 & 1.02 & 0.43 & 0.67 & 0.19 & 0.22 & 0.34 & 1.09 & 0.74 & 2.14 & 0.13 & 0.15 & 0.08 & 0.07 \\
\hline \multicolumn{17}{|c|}{ Nutrient management } \\
\hline $\mathrm{F}_{1}$ & 51.3 & 54.4 & 52.6 & 53.8 & 4.91 & 5.12 & 4.21 & 4.32 & 16.7 & 17.2 & 42.9 & 44.0 & 2.91 & 2.97 & 4.29 & 4.36 \\
\hline $\mathrm{F}_{2}$ & 55.1 & 59.5 & 55.9 & 55.8 & 5.36 & 5.70 & 4.45 & 4.47 & 18.0 & 18.9 & 45.3 & 45.5 & 3.20 & 3.32 & 4.51 & 4.53 \\
\hline $\mathrm{F}_{3}$ & 58.7 & 63.0 & 60.9 & 61.2 & 5.91 & 6.24 & 4.74 & 4.78 & 19.5 & 20.3 & 49.5 & 49.9 & 3.53 & 3.64 & 4.82 & 4.87 \\
\hline $\mathrm{F}_{4}$ & 63.1 & 67.4 & 65.0 & 65.7 & 6.47 & 6.81 & 5.01 & 5.08 & 21.3 & 22.1 & 52.3 & 53.0 & 3.79 & 3.87 & 5.12 & 5.14 \\
\hline SEm \pm & 0.33 & 0.93 & 0.06 & 0.28 & 0.11 & 0.17 & 0.05 & 0.05 & 0.07 & 0.26 & 0.21 & 0.65 & 0.03 & 0.03 & 0.02 & 0.01 \\
\hline $\mathrm{CD}(\mathrm{P}=0.05)$ & 0.97 & 2.78 & 0.19 & 0.85 & 0.32 & 0.52 & 0.14 & 0.16 & 0.22 & 0.76 & 0.62 & 1.96 & 0.08 & 0.10 & 0.06 & 0.04 \\
\hline
\end{tabular}




\section{Int.J.Curr.Microbiol.App.Sci (2017) 6(11): 2405-2415}

Table.3 Effect of irrigation regimes, mulching and integrated nutrient management on N, P K and S uptake

\begin{tabular}{|c|c|c|c|c|c|c|c|c|}
\hline \multirow[t]{2}{*}{ Treatment } & \multicolumn{2}{|c|}{$\begin{array}{l}\text { Total uptake of } \\
\mathrm{N}\left(\mathrm{kg} \mathrm{ha}^{-1}\right)\end{array}$} & \multicolumn{2}{|c|}{$\begin{array}{l}\text { Total uptake of } \\
\mathrm{P}\left(\mathrm{kg} \mathrm{ha}^{-1}\right)\end{array}$} & \multicolumn{2}{|c|}{$\begin{array}{l}\text { Total uptake of } \\
\mathrm{K}\left(\mathrm{kg} \mathrm{ha}^{-1}\right)\end{array}$} & \multicolumn{2}{|c|}{$\begin{array}{l}\text { Total uptake of } \\
\mathrm{S}\left(\mathrm{kg} \mathrm{ha}^{-1}\right)\end{array}$} \\
\hline & 2015 & 2016 & 2015 & 2016 & 2015 & 2016 & 2015 & 2016 \\
\hline \multicolumn{9}{|l|}{ Irrigation regimes } \\
\hline Irrigation at $60 \mathrm{~mm} \mathrm{CPE}$ & 116.9 & 123.1 & 10.83 & 11.39 & 66.9 & 69.1 & 8.18 & 8.42 \\
\hline Irrigation at $80 \mathrm{~mm} \mathrm{CPE}$ & 116.1 & 119.8 & 10.35 & 10.64 & 66.6 & 67.6 & 8.07 & 8.15 \\
\hline Irrigation at $100 \mathrm{~mm} \mathrm{CPE}$ & 113.9 & 117.5 & 9.57 & 9.84 & 65.3 & 66.2 & 7.91 & 8.00 \\
\hline $\mathrm{SEm} \pm$ & 0.99 & 1.07 & 0.24 & 0.34 & 0.15 & 0.20 & 0.09 & 0.09 \\
\hline $\mathrm{CD}(\mathrm{P}=0.05)$ & 2.96 & 3.21 & 0.70 & 1.00 & 0.46 & 0.59 & 0.26 & 0.28 \\
\hline \multicolumn{9}{|l|}{ Mulch } \\
\hline Paddy straw mulch (10 t ha $\left.{ }^{-1}\right)$ & 116.4 & 124.0 & 10.31 & 10.85 & 66.7 & 69.8 & 8.19 & 8.42 \\
\hline Dust mulch & 114.8 & 116.1 & 10.19 & 10.39 & 65.9 & 65.4 & 7.90 & 7.96 \\
\hline $\mathrm{SEm} \pm$ & 0.53 & 0.70 & 0.20 & 0.14 & 0.12 & 0.15 & 0.08 & 0.06 \\
\hline $\mathrm{CD}(\mathrm{P}=0.05)$ & 1.56 & 2.10 & NS & 0.45 & 0.37 & 0.46 & 0.25 & 0.19 \\
\hline \multicolumn{9}{|l|}{ Nutrient management } \\
\hline $100 \%$ NPK & 103.8 & 108.1 & 9.10 & 9.43 & 59.5 & 61.1 & 7.21 & 7.34 \\
\hline $75 \% \mathrm{NPK}+25 \% \mathrm{~N}$ through FYM+ $20 \mathrm{~kg} \mathrm{~S}$ through gypsum & 110.9 & 115.2 & 9.79 & 10.16 & 63.2 & 64.3 & 7.72 & 7.86 \\
\hline $75 \% \mathrm{NPK}+25 \% \mathrm{~N}$ through vermicompost $+40 \mathrm{~kg} \mathrm{~S}$ through gypsum & 119.5 & 124.1 & 10.63 & 11.01 & 68.9 & 70.1 & 8.36 & 8.52 \\
\hline $75 \% \mathrm{NPK}+25 \% \mathrm{~N}$ through FYM + $60 \mathrm{~kg} \mathrm{~S}$ through gypsum & 128.0 & 133.0 & 11.46 & 11.88 & 73.5 & 75.0 & 8.92 & 9.02 \\
\hline $\mathrm{SEm} \pm$ & 0.32 & 0.31 & 0.16 & 0.12 & 0.09 & 0.13 & 0.05 & 0.04 \\
\hline $\mathrm{CD}(\mathrm{P}=0.05)$ & 0.96 & 0.92 & 0.45 & 0.37 & 0.26 & 0.38 & 0.17 & 0.11 \\
\hline
\end{tabular}


Int.J.Curr.Microbiol.App.Sci (2017) 6(11): 2405-2415

Table.4 pH, EC and bulk density after harvest as influenced by irrigation regimes, mulching, integrated nutrient management on summer groundnut

\begin{tabular}{|c|c|c|c|c|c|c|c|c|c|c|}
\hline \multirow[t]{2}{*}{ Treatments } & \multicolumn{2}{|l|}{$\mathrm{pH}$} & \multicolumn{2}{|c|}{$\mathrm{EC}\left(\mathrm{dS} \mathrm{m}{ }^{-1}\right)$} & \multicolumn{2}{|c|}{ Organic carbon $(\%)$} & \multicolumn{2}{|c|}{$\begin{array}{l}\text { Kernel yield (kg } \\
\left.\mathrm{ha}^{-1}\right)\end{array}$} & \multicolumn{2}{|c|}{$\begin{array}{l}\text { Pod yield ( } \mathrm{kg} \mathrm{ha} \\
1 \text { ) }\end{array}$} \\
\hline & 2015 & 2016 & 2015 & 2016 & 2015 & 2016 & 2015 & 2016 & 2015 & 2016 \\
\hline \multicolumn{11}{|l|}{ Irrigation regimes } \\
\hline Irrigation at $60 \mathrm{~mm} \mathrm{CPE}$ & 7.85 & 7.78 & 0.188 & 0.178 & 0.354 & 0.360 & 1839 & 2038 & 4070 & 4232 \\
\hline Irrigation at $80 \mathrm{~mm} \mathrm{CPE}$ & 7.86 & 7.79 & 0.187 & 0.175 & 0.352 & 0.356 & 1796 & 1935 & 3998 & 4110 \\
\hline Irrigation at $100 \mathrm{~mm} \mathrm{CPE}$ & 7.86 & 7.79 & 0.188 & 0.177 & 0.350 & 0.352 & 1715 & 1863 & 3910 & 3995 \\
\hline $\mathrm{CD}(\mathrm{P}=0.05)$ & NS & NS & NS & NS & NS & NS & 31.4 & 35.3 & 11.9 & 18.5 \\
\hline \multicolumn{11}{|l|}{ Mulching } \\
\hline Paddy straw mulch $\left(10 \mathrm{t} \mathrm{ha}^{-1}\right)$ & 7.85 & 7.78 & 0.182 & 0.172 & 0.353 & 0.359 & 1827 & 2051 & 4010 & 4238 \\
\hline Dust mulch & 7.86 & 7.80 & 0.197 & 0.190 & 0.350 & 0.354 & 1740 & 1840 & 3976 & 3987 \\
\hline $\mathrm{CD}(\mathrm{P}=0.05)$ & NS & NS & NS & NS & NS & NS & 25.6 & 28.8 & 9.8 & 15.1 \\
\hline \multicolumn{11}{|l|}{ Nutrient management } \\
\hline $100 \%$ NPK & 7.86 & 7.79 & 0.185 & 0.174 & 0.349 & 0.354 & 3729 & 3895 & 1626 & 1758 \\
\hline $\begin{array}{l}75 \% \mathrm{NPK}+25 \% \mathrm{~N} \text { through } \\
\text { FYM+ } 20 \mathrm{~kg} \mathrm{~S} \text { through } \\
\text { gypsum }\end{array}$ & 7.85 & 7.80 & 0.184 & 0.173 & 0.352 & 0.357 & 3906 & 3987 & 1719 & 1893 \\
\hline $\begin{array}{l}75 \% \mathrm{NPK}+25 \% \mathrm{~N} \text { through } \\
\text { vermicompost+ } 40 \mathrm{~kg} \mathrm{~S} \\
\text { through gypsum }\end{array}$ & 7.85 & 7.80 & 0.184 & 0.173 & 0.351 & 0.355 & 4090 & 4190 & 1836 & 2008 \\
\hline $\begin{array}{l}75 \% \mathrm{NPK}+25 \% \mathrm{~N} \text { through } \\
\text { FYM+ } 60 \mathrm{~kg} \mathrm{~S} \text { through } \\
\text { gypsum }\end{array}$ & 7.86 & 7.79 & 0.186 & 0.175 & 0.353 & 0.360 & 4248 & 4377 & 1952 & 2122 \\
\hline $\mathrm{CD}(\mathrm{P}=0.05)$ & NS & NS & NS & NS & NS & NS & 4.2 & 6.5 & 16.4 & 18.1 \\
\hline Initial Value & 7.89 & 7.82 & 0.186 & 0.176 & 0.355 & 0.361 & - & - & - & - \\
\hline
\end{tabular}


Table.5 Available N, P, K and S after harvest as influenced by irrigation, mulching and integrated nutrient management

\begin{tabular}{|c|c|c|c|c|c|c|c|c|}
\hline Treatments & \multicolumn{2}{|c|}{$\begin{array}{l}\text { Available N (kg ha- } \\
\text { 1) }\end{array}$} & \multicolumn{2}{|c|}{$\begin{array}{l}\text { Available } \mathrm{P}(\mathrm{kg} \mathrm{ha} \\
\left.{ }^{1}\right)\end{array}$} & \multicolumn{2}{|c|}{$\begin{array}{l}\text { Available } \mathrm{K}(\mathrm{kg} \mathrm{ha} \\
1 \text { ) }\end{array}$} & \multicolumn{2}{|c|}{$\begin{array}{l}\text { Available S (kg ha } \\
\text { 1) }\end{array}$} \\
\hline 2015 & & 2016 & 2015 & 2016 & 2015 & 2016 & 2015 & 2016 \\
\hline \multicolumn{9}{|l|}{ Irrigation regimes } \\
\hline Irrigation at $60 \mathrm{~mm} \mathrm{CPE}$ & 197.8 & 201.4 & 16.1 & 17.8 & 230.2 & 232.3 & 14.5 & 14.7 \\
\hline Irrigation at $80 \mathrm{~mm} \mathrm{CPE}$ & 199.7 & 202.7 & 16.8 & 19.1 & 231.6 & 234.7 & 16.0 & 16.2 \\
\hline Irrigation at $100 \mathrm{~mm} \mathrm{CPE}$ & 200.7 & 203.4 & 17.6 & 19.7 & 232.8 & 235.6 & 16.2 & 16.8 \\
\hline $\mathrm{SEm} \pm$ & 0.65 & 0.45 & 0.21 & 0.35 & 0.74 & 0.48 & 0.07 & 0.08 \\
\hline $\mathrm{CD}(\mathrm{P}=0.05)$ & 1.95 & 1.36 & 0.63 & 1.07 & 2.20 & 1.42 & 0.23 & 0.25 \\
\hline \multicolumn{9}{|l|}{ Mulch } \\
\hline Paddy straw mulch $\left(10 \mathrm{t} \mathrm{ha}^{-1}\right)$ & 198.1 & 201.3 & 16.4 & 17.9 & 229.9 & 232.5 & 15.1 & 15.5 \\
\hline Dust mulch & 200.6 & 203.6 & 17.1 & 19.8 & 233.1 & 235.9 & 16.0 & 16.2 \\
\hline $\mathrm{SEm} \pm$ & 0.58 & 0.40 & 0.19 & 0.31 & 0.66 & 0.43 & 0.06 & 0.07 \\
\hline $\mathrm{CD}(\mathrm{P}=0.05)$ & 1.74 & 1.21 & 0.56 & 0.95 & 1.96 & 1.27 & 0.21 & 0.22 \\
\hline \multicolumn{9}{|l|}{ Nutrient managemet } \\
\hline $100 \%$ NPK & 196.6 & 199.2 & 16.4 & 18.3 & 230.1 & 233.4 & 13.7 & 14.9 \\
\hline $75 \% \mathrm{NPK}+25 \% \mathrm{~N}$ through FYM+ $20 \mathrm{~kg} \mathrm{~S}$ through gypsum & 198.7 & 200.6 & 16.6 & 18.5 & 230.3 & 233.9 & 15.8 & 16.0 \\
\hline $75 \% \mathrm{NPK}+25 \% \mathrm{~N}$ through vermicompost $+40 \mathrm{~kg} \mathrm{~S}$ through gypsum & 200.2 & 204.9 & 16.9 & 19.3 & 232.4 & 234.5 & 16.1 & 16.2 \\
\hline $75 \% \mathrm{NPK}+25 \% \mathrm{~N}$ through FYM+ $60 \mathrm{~kg} \mathrm{~S}$ through gypsum & 202.2 & 205.3 & 17.1 & 19.5 & 233.2 & 234.9 & 16.6 & 16.5 \\
\hline $\mathrm{SEm} \pm$ & 0.46 & 0.16 & 0.08 & 0.11 & 0.20 & 0.19 & 0.02 & 0.03 \\
\hline $\mathrm{CD}(\mathrm{P}=0.05)$ & 1.37 & 0.47 & 0.25 & 0.31 & 0.58 & 0.55 & 0.07 & 0.09 \\
\hline Initial Value & 202.0 & 199.7 & 18.8 & 16.8 & 233.5 & 232.6 & 15.5 & 15.6 \\
\hline
\end{tabular}




\section{Available N, P, K and S in soil}

Increased frequency of irrigation significantly decreased the available $\mathrm{N}, \mathrm{P}, \mathrm{K}$ and $\mathrm{S}$ in soil and it was found to be the highest with the irrigation at $100 \mathrm{~mm} \mathrm{CPE}$. The reason could be that insufficient moisture under this treatment did not support the adequate uptake of $\mathrm{N}, \mathrm{P}, \mathrm{K}$ and $\mathrm{S}$ by crop and most of these nutrients were left in the soil. Results are corroborated with the research findings of Verma et al., (2012). Dust mulch resulted significantly higher available N, P, K and S compared to paddy straw mulch. This is because of lower crop yield and poor utilization of nutrients under this treatment by groundnut. Higher amount of available N, P, $\mathrm{K}$ and $\mathrm{S}$ was recorded with $75 \% \mathrm{NPK}+25 \%$ $\mathrm{N}$ through FYM + $60 \mathrm{~kg} \mathrm{~S}$ through gypsum.

Increase in available $\mathrm{N}, \mathrm{P}, \mathrm{K}$ and $\mathrm{S}$ due to organic manure FYM, vermicompost and chemical fertilizers application may be attributed to the direct addition of these nutrients to the available pool of soil. The favourable effect of organic manure and chemical fertilizers in enhancing the availability of nutrients in soil was also reported by Singh et al., (2006) and Mohapatra and Dixit (2010).

The build-up of soil available nutrients may also be attributed to the addition of FYM and vermicompost for the conversion of organically bound nutrients to inorganic forms and helped in the mineralization of soil nutrients leading to build-up of higher available nutrients. Varalakshmi et al., (2005) had reported increase in $\mathrm{N}$ content of soil with integrated nutrient management in groundnut. Incorporation of farmyard manure, lime and $\mathrm{P}$ fertilizer reduced the fixation of water soluble $\mathrm{P}$ and increased the mineralization of organic $\mathrm{P}$ due to microbial action which resulted in increase in available $\mathrm{P}$ in soil. An increase in $\mathrm{K}$ availability probably was from $\mathrm{K}$ bearing minerals by the organic acids released as a decomposition of FYM as reported by Singh et al., (2006).

\section{Kernel and haulm yield}

The significantly highest kernel and haulm yield was achieved under irrigation at $60 \mathrm{~mm}$ CPE followed by irrigation at $80 \mathrm{~mm}$ and 100 $\mathrm{mm}$ CPE, respectively (Table 4). The better development of crop under irrigated treatments was a result of better moisture availability, which maintained the internal water balance of the plant. Increase in yield is due to the increase in the yield attribute reported by Gupta et al., (2015). Paddy straw mulch recorded significantly higher kernel and haulm yield as compared to dust mulch. The better development of crop under paddy straw mulch treatment was a result of better moisture availability, which maintained the internal water balance of the plant. Increase in yield is due to the increase in the yield attribute reported by Ravisankar et al., (2014). Among INM treatments, an application of $75 \% \mathrm{NPK}+25 \% \mathrm{~N}$ through FYM $+60 \mathrm{~kg} \mathrm{~S}$ through gypsum recorded significantly the highest kernel and haulm yield followed by $75 \% \mathrm{NPK}+25 \% \mathrm{~N}$ through vermicompost + $40 \mathrm{~kg} \mathrm{~S}$ through gypsum as compared to other INM treatments. These results are corroborated with the research results of Vishwakarma et al., (2012), Chavan et al., (2014) and Patil et al., (2017).

\section{References}

Arunachalam, P., Kannan, J., Prabhaharan, G., Kumar, P. and Zadda, K. 2012. Response of groundnut genotypes to soil fertilization of micronutrients in alfisol conditions. Electronic Journal of Plant Breeding 4(1): 1043-1049.

Baliarsingh, A. and Mahapatra, P.K. 2015. Effect of date of sowing and irrigation on yield and moisture use dynamics of 
summer groundnut. Environment and Ecology 33:175-179.

Chaudhary, J.H., Sutaliya, R. and Desai, L.J. 2015. Growth, yield, yield attributes and economics of summer groundnut as influenced by integrated nutrient management. Journal of Applied and Natural Science 7(1): 369-372.

Chavan, A.P. Jain, N.K. Sagvekar, V.V. and Kumar, T. 2014. Integrated nutrient management in groundnut. Research on Crops 15: 454-460.

Choudhary, M. Chovatia, P.K. Jat, R. and Choudhary, S. 2017. Effect of weed management on growth attributes and yield of summer groundnut. International Journal of Chemical Studies 5: 212-214.

Choudhary, S.K., Jat, M. K., Sharma, S.R. and Singh, P. 2011. Effect of INM on soil nutrient and yield in groundnut field of semi-arid area of Rajasthan. Legume Res. 34(4): 283 - 287.

Dutta, D. and Mondal, S. S. 2006. Response of summer groundnut to moisture stress, organic manure and fertilizer with and without gypsum under lateritic soil of West Bengal. Indian Journal of Agronomy 51(2): 145-148.

Gupta, B. Kapil, O and Hovav, R. 2015. The effects of irrigation level and genotype on pod-filling related traits in peanut. Journal of Agricultural Science (Toronto) 7: 169-181.

Mohapatra, A. K. B. and Dixit, L. 2010. Integrated nutrient management in rainy season groundnut. Indian Journal of Agronomy 55(2):123-127.

Monsefi, A., Sharma, A.R. and Zan, N.R. 2016. Weed management and conservation tillage for improving productivity, nutrient uptake and profitability of wheat in soybean -wheat cropping system. J. Agr. Sci. Tech. 18: 411-421.

Noman, H.M., Rana, D.S. and Rana, K.S.
2015. Influence of sulphur and zinc levels and zinc solubilizer on productivity, economics and nutrient uptake in groundnut. Indian Journal of Agronomy 60(2): 301-306.

Noman, H.M., Rana, D.S., Choudhary, A.K., Rajpoot, S. and Paul, T. 2016. Sulphur and $\mathrm{Zn}$ management in groundnutwheat cropping system: direct effects on system productivity and residual effects on yield, energetic and $\mathrm{Zn}$ biofortification in wheat. Indian Journal of Agricultural Sciences 86(4): 441-447.

Patil, D.B. Pawar, P.P. Wadile, S.C. and Patil, H.M. 2017. Effect of integrated nutrient management on growth and yield of kharif groundnut. International journal of Horticulture, Agriculture and Food science 1:21-23.

Patra, Partha, S., Sinha A.C. and Mahesh, S.S. 2011. Yield, nutrient uptake and quality of groundnut kernels as affected by organic sources of nutrient. Ind. J. Agron. 56(3):237-241.

Ravisankar, N., Balakrishnan, M., Ambast, S. K., Srivastava, R. C., Bommayasamy, N. and Subramani, T. 2014. Influence of irrigation and crop residue mulching on yield and water productivity of table purpose groundnut in humid tropical Island. Legume Res. 37 (2): 195-200.

Saha, D. and Gunri, S.K. 2014. Effect of polythene mulching, irrigation regimes and fertilizer dise on summer groundnut nodulation, pod yield and nitrogen availability in soil. Environment and Ecology 32:1301-1303.

Singh, S., Singh, R. N., Prasad, J. and Singh, B. P. 2006. Effect of integrated nutrient management on yield and uptake of nutrients by rice and fertility in rainfed uplands. Journal of the Indian Society of Soil Science 54(3): 327-330.

Sounda, G., Mandal, A. and Moinuddin, G. 2006. Effect of irrigation and mulch on nodulation and yield of summer 
groundnut. Journal of Interacademicia. 10(4): 489-496.

Thiyagarajan, G., Ranghaswamy, M.V., Rajkumar, D. and Kumaraperumal, R. 2010. Deficit irrigation effects on groundnut with micro sprinklers. Madras Agriculture Journal 97:40-42.

Varalakshmi, L.R., Srinivasa, M.C.A. and

Bhaskar, S. 2005. Effect of integrated use of organic manures and inorganic fertilisers on organic carbon, available $\mathrm{N}, \mathrm{P}, \mathrm{K}$ in sustaining productivity of groundnut-finger millet cropping system. Journal of the Indian Society of Soil Science 53(3):315-318.

Verma, S.K. and Idnani, L.K. 2012. Water use, nutrient content and their uptake as influenced by irrigation and nitrogen management in potato-maize cropping system. Indian Journal of Agricultural Sciences 82 (12): 1032-1038.

Verma, S.K. Kumar, A. and Idnani, L.K. 2013. Effect of irrigation and nitrogen management on growth and yield of potato and their residual effect on succeeding maize under potato-maize cropping system. Indian Journal of Agronomy 58:48-53.

Verma, S.K., Singh, S.B., Prasad, S.K., Meena, R.N. and Meena, R.S. 2015. Influence of irrigation regimes and weed management practices on water use and nutrient uptake in wheat. Bangladesh J. Bot. 44(3): 437-442.

Vishwakarma, A.K. Brajendra; Pathak, K.A. and Ramakrishna, Y. 2012. Effect of different sources of nutrient application on productivity, nutrient uptake and economics of groundnut in Kolasib district of Mizoram Indian. Journal of Soil Conservation 40: 152-157.

Yadav, G.S., Datta, M., Babu, S., Saha, P. and Singh, R. 2015. Effect of sources and levels of phosphorus on productivity, economics, nutrient acquisition and phosphorus-use efficiency of groundnut under hilly ecosystems of North-East India. Indian Journal of Agronomy 60 (2): 307-311.

\section{How to cite this article:}

Avinash Chandra Maurya, S.K. Verma, Sushil Kumar and Kairovin Lakra. 2017. Nutrient Concentration and their Uptake and Available Nutrients in Soil Influenced by Irrigation, Mulching and Integrated Nutrient Management in Summer Groundnut. Int.J.Curr.Microbiol.App.Sci. 6(11): 2405-2415. doi: https://doi.org/10.20546/ijcmas.2017.611.285 